\title{
The "Myth" of the Minimum SAR Antenna Area Constraint
}

\author{
A. Freeman', W.T.K. Johnson', B. Huneycutt', R. Jordan', S. Hensley', P. Siqueira', J. Curlander' \\ 1. Jet Propulsion Laboratory, California Institute of Technology \\ 4800 Oak Grove Drive, Pasadena, CA 91109 \\ Tel: (818) 3541887 Fax: (818) 3935285 \\ e-mail: tony.freeman@jpl.nasa.gov \\ 2. Vexcel Corporation, Boulder, $\mathrm{CO}$
}

\section{ABSTRACT}

A design constraint traceable to the early days of spaceborne Synthetic Aperture Radar (SAR) is known as the minimum antenna area constraint for SAR. In this paper, it is confirmed that this constraint strictly applies only to the case where both the best possible resolution and the widest possible swath are the design goals. SAR antennas with area smaller than the constraint allows are shown to be possible, have been used on spaceborne SAR missions in the past, and should permit further, lower-cost SAR missions in the future.

\section{INTRODUCTION}

The SAR designer has many parameters to select in specifying a SAR system design, one of which is the antenna size (height and width). A constraint that is often used by SAR designers to belp select these parameters is known as the Minimum SAR Antenna Area Constraint. This constraint states that antennas used in SAR systems must have a certain minimum area for the design to be viable. It is derived in many of the standard texts on the subject (e.g. [1], [2], [3]. [4] and [5]) via a thorough treatment of a special case of SAR design, for which the best possible resolution and the widest possible swath are the design goals. It is clear in the derivation given in [1], for example, that the constraint in question applies only "for realization of full resolution SAR". In this paper, this constraint is examined and shown to apply only in the special case referred to. A more general treatment is also offered here, in which it is shown that smaller SAR antennas are practicable and offer the SAR system designer a greater degree of freedom in system design. This result rests on three insights into spaceborne SAR design that have each been implemented successfully: the selection of a pulse repetition frequency (PRF) smaller than the nominal Doppler bandwidth; the adoption of a smaller processing bandwidth; and the selection of a data window size in range that is less than the illuminated swath.

\section{THEORY}

The geometry under consideration is shown in Figure 1. A planar SAR antenna of length $L_{2}$, and height $W_{2}$, traveling along a straight line trajectory at speed $V$, is pointed in a sidelooking direction, perpendicular to the flight track, so that it illuminates a swath on the ground of width $W_{\text {(max) }}$. This illuminated swath width is determined by the beamwidth of the antenna in the elevation plane and the geometry of the situation, as follows:

$$
\mathrm{W}_{\mathrm{g}(\max )} \approx \frac{\theta_{\mathrm{el}} \mathrm{R}_{\mathrm{m}}}{\cos \eta}=\frac{\lambda \mathrm{R}_{\mathrm{m}}}{\mathrm{W}, \cos \eta}
$$

where the well-known expression for the 3-dB beamwidth of a planar array, i.e. $\theta_{\mathrm{el}}=\lambda / \mathrm{W}_{\mathrm{a}}$, has been used. $W_{(\max )}$ represents the widest possible swath in ground range (or cross-track) for which data can be collected, given an antenna of a particular size and a certain illumination geometry.

Another result well-known to SAR designers is the limiting resolution in the azimuth (or along-track) dimension, given by:

$$
\delta \mathrm{x} \geq \mathrm{L}_{\mathrm{a}} / 2
$$

which simply states that the best possible azimuth resolution that can be achieved for a non-squinting, side-looking SAR with an antenna of length $L_{2}$, is half that antenna length.

Because SARs are pulsed radar systems, the SAR designer's task is complicated by the noed to consider ambiguous returns in both the azimuth and range dimensions. One basic requirement, adapting the arguments given in [1], is that the time of reception of the earliest possible echo from any point within the desired swath due to a particular pulse transmission must be later than the time of reception of the last possible echo from any other point within the illuminated swath due to transmission of the previous pulse. This avoids 


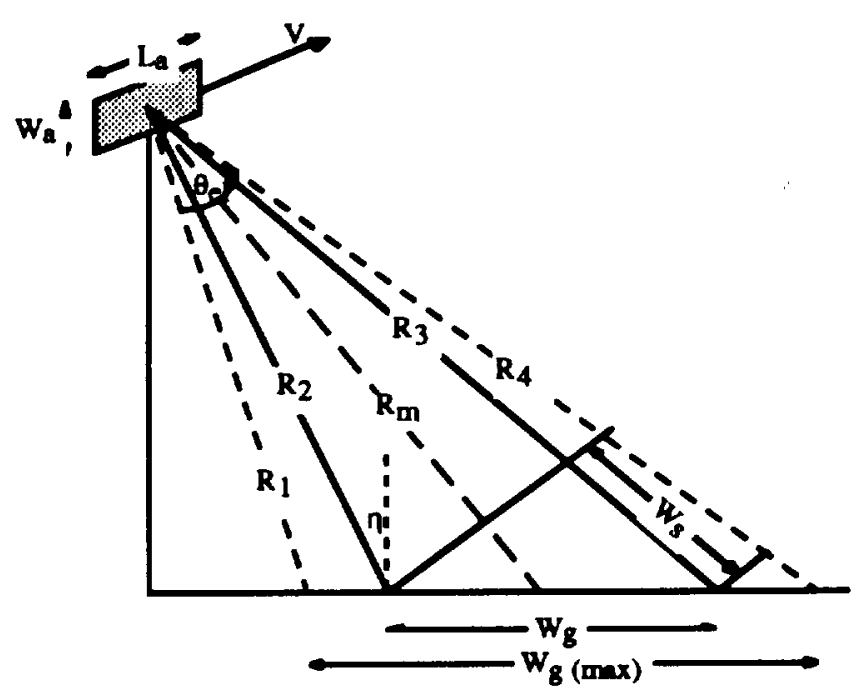

Figure 1: Simplified SAR geometry, showing the swath Illuminated on the ground, $W_{g_{\max }}$, which is determined by the antenna beamwidth, $\theta_{\text {si}}$; and $W_{\text {, }}$, the actual swath for which returns are recorded.

ambiguous returns in range from the main lobe of the antenna (in elevation) occurring within the desired swath. From Figure 1 , this means that:

$$
2 \mathrm{R}_{4} / \mathrm{c}<2 \mathrm{R}_{2} / \mathrm{c}+\mathrm{IPP}
$$

where $R_{2}$ and $R_{4}$ are the near range limit of the desired swath in slant range and the far range limit of the illuminated swath respectively, and the interpulse period (IPP) is the inverse of the Pulse Repetition Frequency, i.e. IPP $=1 /$ PRF. Note that (3) assumes that the transmitted pulse length is significantly smaller than either of the two path lengths. It is straightforward to incorporate the pulse length into the expression if this is not the case.

Given (3), the width of the desired swath in slant range, $W_{\text {, }}$, is bounded by:

$$
W_{s} \leq\left(R_{4}-R_{2}\right)<c \text { IPP } / 2=c /(2 P R F)
$$

The case $R_{1}=R_{2}$ and $R_{3}=R_{4}$ is of interest, since the desired swath and the illuminated swath are the same. Note that the desired swath need not be as large as the widest possible swath, i.e. the illuminated swath. Thus the radar designer can choose to recond data from a swath smaller than that illuminated on the ground and nood only consider range ambiguities which impact the desired swath. The near range limit of the desired swath can be anywhere within the bounds of the illuminated swath. The far range limit of the desired swath can be anywhere between $R_{2}$ and $R_{4}$. Equation (4) is often expressed as an upper bound on the PRF, i.e.,

$$
P R F<c / 2\left(R_{4}-R_{2}\right)
$$

Thus the smaller the distance between $R_{2}$ and $R_{4}$, the larger the PRF is allowed to be. For a desired swath width smaller than the illuminated swath, the optimum would be to select $\mathbf{R}_{\mathbf{1}}<\mathbf{R}_{\mathbf{2}}$ and $\mathbf{R}_{\mathbf{3}}=\mathbf{R}_{\mathbf{4}}$.

In the azimuth dimension, again after [1], the requirement is to measure Doppler frequency unambiguously over the range of frequencies neded to achieve resolution $\delta x$. This paces a lower bound on the PRF given by:

$$
\text { PRF }>\text { V } / \delta x
$$

In practice the PRF must be significantly greater than this lower bound to avoid aliasing within the processing bandwidth (V/Sx) required to achieve the noeded azimuth resolution. In the limit provided when the best possible resolution is required, as in equation (2), this lower bound becomes:

$$
\text { PRF }>2 \mathrm{~V} / \mathrm{L}_{\mathrm{a}}
$$

which states that the PRF in this case should be greater than the range of Doppler frequencies within the bounds of the area illuminated by the physical antenna in azimuth, which is the Doppler bandwidth for that length of antenna. Note that, for a desired resolution which is worse than the theoretical best possible, equation (6) allows the radar designer to select a PRF which is smaller than the Doppler bandwidth associated with the given length of the antenna. Also, again from [1], the azimuth ambiguities need only be evaluated over the processing bandwidth required to achieve the needed azimuth resolution, not over the entire range of frequencies which the PRF spans.

Combining the constraints given in (4) and (6) yields:

$$
\mathrm{W}_{\mathrm{s}}<\frac{\mathrm{c}}{2 \mathrm{PRF}}<\frac{\mathrm{c}}{2 \mathrm{~V}} \delta \mathrm{x}
$$

which, as noted in [1], requires that the swath width $W_{1}$ decrease as the azimuth resolution $\delta x$ improves (i.e. becomes smaller). Rearranging (8), the relationship between (slant range) swath width and (azimuth) resolution can be more clearly seen,

$$
\frac{W_{s}}{\delta x}<\frac{c}{2 V}
$$

which is a well-known result [1]. For Low Earth Orbit satellites, $c / 2 V$ is nearly constant (at 20,000). For airbome systems, $c / 2 \mathrm{~V}$ is typically in the range 300,000 to 750,000 and satisfying the constraint given in (9) is rarely a problem. 
The swath width in slant range can be related to the swath width in ground range via the nominal relation:

$$
w_{s}=w_{g} \sin \eta
$$

[which is easily generalizable to the case for wide swath SARs, for which $\eta$ varies significantly across the swath.] Using equations (1) and (2), combined with (10), in equation (9), the constraint for the case when both the best possible resolution and the widest possible swath are required, can be obtained:

$$
\frac{W_{s(\max )}}{\delta x_{(\min )}}=2 \frac{\lambda R_{m}}{W_{a}} \frac{\tan \eta}{L_{a}}<\frac{c}{2 V}
$$

So the antenna area is restricted in this case by:

$$
A_{a}=W_{a} L_{a}>\frac{4 V \lambda R_{m}}{c} \tan \eta
$$

which is a form of the commonly used minimum antenna area constraint for SARs. SAR system designers often introduce an additional design margin on top of this, so that the actual area of the antenna is given by:

$$
A_{\mathrm{a}}=\frac{\mathrm{K} 4 \mathrm{~V} \lambda \mathrm{R}_{\mathrm{m}}}{\mathrm{c}} \tan \eta
$$

where $K$ is in the range 1 to 3 .

As is clear from the above, equations (12) and (13) only apply to a special case, which is when the radar designer seeks to achieve both the best possible resolution and the widest possible swath at the same time. The fundamental constraint is actually given in equation (9), which places a limit on the ratio of the swath width versus azimuth resolution that really only depends on the platform speed $V$.

\section{DISCUSSION}

The derivation above shows that there is no need to constrain SAR antennas to be a certain minimum area. In particular, when designing a SAR system which does not have to achieve both the best possible resolution and the best possible swath width at the same time the SAR system designer is free to select a smaller antenna than would be the case for a SAR optimized to achieve these goals. This has significant impact on the design of multi-mode SARs, such as NASA's proposed LightSAR instrument, which may be optimized for one mode but not another, and in the design of moderate resolution SARs, which may take advantage of nonplanar antennas, and other antennas which are not optimized for SAR performance but which may be more cost effective. An excellent example of the latter was the Magellan SAR design [6], which took an existing $3.7 \mathrm{~m}$ diameter parabolic reflector antenna designed for communications and not optimized for SAR data collection, and successfully imaged $97 \%$ of the surface of Venus at $100-300 \mathrm{~m}$ resolution. Another example was SIR-B, which successfully collected data at a look angle of 60 degrees, though at that angle the antenna area was only half that specified by (12).

This does not mean that SAR antennas can be arbitrarily small in size. The size of the antenna has significant impact on the gain and therefore on the signal-to-noise ratio which must be taken into account. The analysis presented in this paper is no substitute for a rigorous treatment of the calculation of range and azimuth ambiguity levels, which must be factored in by the designer. The exact form of the antenna pattern and other radar parameters such as range, PRF, processing bandwidth, and the radar backscatter as a function of incidence angle must all be incorporated into such a calculation.

\section{ACKNOWLEDGMENTS}

The research described in this paper was carried out by the Jet Propulsion Laboratory, California Institute of Technology, under a contract with the National Aeronautics and Space Administration.

\section{REFERENCES}

[1] Curlander, J. C. and McDonough, R. N., "Synthetic Aperture Radar. Systems and Signal Processing", publ. John Wiley, 1991

[2] Ulaby, F. T., Moore, R. K. and Fung, A. K., "Microwave Remote Sensing: Active and Passive", Vol. II, publ. Addison-Wesley, 1982.

[3] Elachi, C. "Spaceborne Radar Remote Sensing: Applications and Techniques", publ. IEEE Press, 1988.

[4] McCandless, S. W., Jr., "SAR in Space - The Theory, Design, Engineering and Application of a Space-Based SAR system", chapter 4 of "Space-Based Radar Handbook', Cantafio, L. J. (ed.), publ. Artech House, 1989.

[5] Raney, R. K., "Radar Fundamentals: Technical Perspective", in Henderson, F. M. and Lewis, A. J., 'Principles and Applications of Imaging Radar', Vol. 2 of Manual of Remote Sensing Third Edition (ed. R. A. Reyerson), Third Edition, publ. John Wiley \& Sons, 1998.

[6] Johnson, W. T., "Magellan Imaging Radar Mission to Venus", Proc. IEEE, Vol. 79, No. 6, June 1991, pp. 777-790, 1991. 